EPJ Web of Conferences 43, 05004 (2013)

DOI: $10.1051 /$ epjconf/20134305004

(C) Owned by the authors, published by EDP Sciences, 2013

\title{
Ages of evolved low mass stars: Central stars of planetary nebulae and white dwarfs
}

\author{
W.J. Maciel ${ }^{\mathrm{a}}$, T.S. Rodrigues and R.D.D. Costa \\ Astronomy Department, University of São Paulo, Rua do Matão 1226, \\ CEP 05508-090 São Paulo SP, Brazil
}

\begin{abstract}
We have developed several methods to estimate the ages of central stars of planetary nebulae (CSPN), which are based either on observed nebular properties or on data from the stars themselves. Our goal is to derive the age distribution of these stars and compare the results with empirical distributions for CSPN and white dwarfs. We have initially developed three methods based on nebular abundances, using (i) an age-metallicity relation which is also a function of the galactocentric distance; (ii) an age-metallicity relation obtained for the galactic disk, and (iii) the central star masses derived from the observed nitrogen abundances. In this work we present two new, more accurate methods, which are based on kinematic properties: (I) in this method, the expected rotation velocities of the nebulae around the galactic centre at their galactocentric distances are compared with the predicted values for the galactic rotation curve, and the differences are attributed to the different ages of the evolved stars; (II) we determine directly the U, V, $\mathrm{W}$, velocity components of the stars, as well as the velocity dispersions, and use the dispersion-age relation by the Geneva-Copenhagen survey. These methods were applied to two large samples of galactic CSPN. We conclude that most CSPN in the galactic disk have ages under 5 Gyr, and that the age distribution is peaked around 1 to $3 \mathrm{Gyr}$.
\end{abstract}

\section{INTRODUCTION}

Why do we need stellar ages? The main motivation of this work is the study of the time variation of the abundance gradients in the galactic disk. Some recent theoretical models predict a time flattening of the observed radial abundance gradients, while other models predict the opposite behaviour. This can be analyzed on the basis of abundances of planetary nebulae and open cluster stars, and in both cases the results depend on the ages of the objects considered. CSPN and white dwarfs are the offspring of intermediate mass stars with main sequence masses between 0.8 and 8 solar masses, approximately. As a consequence, their properties reflect different physical conditions depending on the stellar masses, and therefore ages, which makes these objects extremely important in the study of the chemical evolution of the Galaxy. The determination of ages is a complex problem, and there is presently no single method that can be generally applied. We have developed several methods to estimate the ages of the PN progenitor stars, based both on the observed nebular properties and in some properties of the stars themselves (see [1] and [2] for a detailed discussion). In this work, we discuss two kinematic methods based on the age-velocity dispersion relation obtained by the Geneva-Copenhagen survey.

\section{AGE DETERMINATION OF CSPN}

Method I - Knowing the stellar radial velocities $V_{r}$ (LSR) and distances $d$, it is possible to derive the expected rotation velocity at the galactocentric position of the nebula, $\Theta(R)$. Adopting a standard

\footnotetext{
a e-mail: maciel@astro.iag.usp.br
}

This is an Open Access article distributed under the terms of the Creative Commons Attribution License 2.0, which permits unrestricted use, distribution, and reproduction in any medium, provided the original work is properly cited. 


\section{EPJ Web of Conferences}

rotation curve $\Theta_{c}(R)$, the difference between the measured and expected velocities can be derived and related to the stellar age $t$, neglecting other effects that may affect the velocities. Using the velocity dispersion-age relation obtained by the Geneva-Copenhagen survey [3] [4] [5], the age distribution of the CSPN can be obtained. In Method I we have used both the $\mathrm{V}$ and the total $\mathrm{T}$ velocity components. Method II - This method does not involve any assumptions on the rotation curve, but consists in calculating the $\mathrm{U}, \mathrm{V}, \mathrm{W}$, and total $\mathrm{T}$ velocity components from the CSPN data, namely, distances, radial and tangential velocities and proper motions. We have adopted the following hypotheses: (i) the tangential velocities are similar to the radial velocities in average; (ii) in view of the large distances of the nebulae, the proper motions were neglected in a first approximation; (iii) as a second approximation, the proper motions were taken into account, assuming that the motion in right ascension is similar to the motion in declination, in average. These hypotheses were confirmed by a comparison with the objects in the samples having measurements of these quantities.

\section{RESULTS AND DISCUSSION}

We have considered two large samples of galactic CSPN containing 234 well known nebulae (Sample 1) and 867 objects with accurate radial velocities (Sample 2), respectively. It can be shown that the results are very similar in both cases. They are not affected by the adopted distance scale, the adopted rotation curve, or the velocity component considered. Most stars are located in the first few bins, so that their ages are expected to be within $3 \mathrm{Gyr}$, approximately. Results for Method II are very similar to those of Method I. In both methods most stars have ages under $5 \mathrm{Gyr}$, as in the previous methods discussed by [1], but the derived ages from the two kinematic methods discussed here are somewhat lower, concentrating in the first few age bins. Again the results do not depend on the adopted distance scale, on the considered velocity component $(\mathrm{U}, \mathrm{V}, \mathrm{W}, \mathrm{T})$, or on the hypotheses concerning the proper motions and tangential velocities. However, the choice of the lower age bracket in the velocity dispersion-age relation may affect the population of the initial bin, which includes objects with ages under 1 Gyr, approximately. Adopting a limit of about $0.5 \mathrm{Gyr}$, the population of the first bin decreases, but the main conclusion is maintained, namely, most objects still have ages under 3 Gyr approximately.

We have also compared the obtained distributions with the expected age distributions of CSPN based on empirical masses and that of white dwarfs based on their better known mass distributions. Using standard mass-age relations, the agreement of both distributions is very good, confirming that the duration of the PN phase is short compared to the total age of an intermediate mass star.

\section{References}

[1] Maciel, W.J., Costa, R.D.D., Idiart, T.E.P., A\&A, 512, (2010) A19

[2] Maciel, W.J., Rodrigues, T.S., Costa, R.D.D., Rev. Mex. A\&A, 47, (2011) 401

[3] Nordström, B., Mayor, M., Andersen, J. et al., A\&A, 418, (2004) 989

[4] Holmberg, J., Nordström, B., Andersen, J., A\&A, 475, (2007) 519

[5] Holmberg, J., Nordström, B., Andersen, J., A\&A, 501, (2009) 941 